\title{
Identification and Classification of Rice Plant Diseases using Machine Learning
}

\author{
Jyoti Dinkar Bhosale \\ Department of Computer \\ Engineering, \\ Shri Jagdishprasad Jhabarmal \\ Tibrewala University, \\ Jhunjhunu, Rajasthan
}

\author{
Lomte Santosh S., PhD \\ Department of Computer \\ Engineering, \\ Shri Jagdishprasad Jhabarmal \\ Tibrewala \\ University, Jhunjhunu, Rajasthan
}

\author{
Prasadupeedi, $\mathrm{PhD}$ \\ Department of Computer \\ Engineering, \\ Shri Jagdishprasad Jhabarmal \\ Tibrewala University, \\ Jhunjhunu, Rajasthan
}

\begin{abstract}
In this paper Plant disease identification is crucial for preventing reductions in agricultural output quantity and production. To ease agricultural issues, several machine learning and image processing technologies are applied. This review is mostly concerned with rice plant disease. Detection based on image inputs from ill rice plants using various ML and image processing algorithms. Furthermore, the important ML and image processing concepts in plant identification and categorization there has been mention of sicknesses. Probabilistic Neural Network (PNN), Evolutionary Techniques (GA), \& k-Nearest Neighbor (K-Nearest Neighbor) are three classification algorithms. Neighbor Classifier (KNN) \& Support Vector Machine (SVM) are two more (SVM). The reliability of an output relies on the input data when used in a number of agricultural research applications. As a consequence, selecting a categorizing approach is a major duty. Agriculture, biological research, and so forth. Are there other industries that employ leaf disease classification? Comprehensive research into rice plant diseases, image dataset size, processing \& segmentation methodologies, or classifiers are all important variables to consider.
\end{abstract}

\section{Keywords}

Image Processing, Disease Detection, Segmentation, Feature Extraction, Classification, Machine learning, Rice plant diseases, Segmentation

\section{INTRODUCTION}

Agriculture is indeed the principal source of income in many countries across the world. Farmers choose crops, paddies, and pesticides based on the significance of agriculture in order to promote plant growth in a brief amount of time. In many countries, rice is the main food crop.[Shrivastava\& Pradhan, 2021] Rice plants now are facing major issues in the agricultural business as a consequence of diseases that damage quality.[Kornacki, 2010] In addition to the quantity of crops, there is a shortage of expert availability of the agricultural region, and a lack of knowledge in the many factors for the lower yield, which include fertiliser management or a lack of data about pest and diseases. Production rate.[Sandhu\& Kaur, n.d.] Plant diseases may have an indirect and direct influence on the environment. Diseases spread as a result, causing damage to the tree's overall functioning and damage to the environment. By dramatically lowering the number of crops grown, you may improve your financial status. A variety of fungal and bacterial diseases affect plants. The most prevalent are sheath diseases, NBSD leaf blast, and leaf spots. There are several diseases that damage rice plants.[Edelhoff et al., 2016] The severity of the Blast's devastation may be observed in the damage produced by the Blast. The severity of the condition Bipolarize is also accountable for brown spot, a well-known rice disease. Orzo is a kind of fungus that may be spotted throughout the growing season. Brown patches often are severe. When rice is grown on soils deficient in silicon. Diseases of rice cause a loss of 10 to $15 \%$ of output in Asia. An agricultural professional has recently been directly aiding with analysis and monitoring. Plant ailments need more work or a longer process time.[Saranya\& Mythili, 2020] Farmers are sometimes challenged with difficulties in recognizing diseases, resulting in crop loss. One of the most effective methods an automation process that scans photographs of "supposedly" ill leaves might be beneficial to farmers. The Report Advanced Science - Technical Publication is a participant international journal publishes system. Diseases are identified using the plant leaflets that disclose sickness symptoms. Farmers may be warned in real time about disease outbreaks thanks to automated technologies. Certain crops did not carry fruit. Because the latency in detecting sickness as a consequence, early disease detection is crucial.

\subsection{Image Segmentation}

Segmenting a leaf image includes dividing the picture into discrete segments with same properties. To segment data, Otsu's, k-means clustering, or other methods may be utilized. Thresholding, region and edge-based procedures, and so on are examples of approaches.[International Commission on Microbiological Specifications for Foods (ICMSF), 2011]

\subsection{Feature Extraction}

The extract of characteristics is crucial in the classification of disorders. Plant disease color, texture, and morphology are utilized to extract characteristics in a range of applications. Classification[Kornacki, 2010]. The roughness of a surface describes how the color is distributed. Image, roughness, \& image hardness Morphological Color and texture characteristics, for example, surpass feature extraction. Leaf disease detection

\subsection{Disease Classification}

According to my observations the most challenging challenge in picture analysis is segmentation. The primary purpose of classification is to correctly anticipate the values of a continuous classier given a collection of data. A vector represents predictors or attributes. The classification of plant diseases is based on whether or not the image is diseased.[Gurtler et al., n.d. ] 


\subsection{Image Pre-processing}

Pre-processing procedures, such as picture cropping to change the picture size and shape, image smoothness to eliminate noise, and image enhancement, have been used to enhance image data. Image contrast or color conversion should be improved.[ERKMEN, 2021]

\subsection{Rice Blast (RB)}

In this paper Magnaporthe orate produces RB disease, according to the description. This sickness is characterized by greyish green round dots just on leaves with a deep green border. The lesions are elliptical and spindly in shape.[Mead, 2007]One of them is this. The most dangerous rice diseases are seen in a variety of countries throughout the world. There are a total of 88 photographs of RB.[Shrivastava\& Pradhan, 2021]

\subsection{Objectives of the study}

The following are the study's key objectives in this paper:

- To study various rice leaf diseases.

- To explore classifications for detecting rice disease.

- To apply the Leaf Disease Diagnosis, use the proposed versions of the Disease Detection Diagnosis Systems.

- To study and test different rice leaf diseases on the basis of various images.

- Classify plant leaf diseases into three distinct groups (leaf blast, leaf blight, and brownspot).

- Increase the Accuracy of Predictions.

\subsection{Scope of the study}

- Identify an illness or its underlying cause.

- To avoid crop loss.

\subsection{Limitations of the study}

- This section discusses my preferred rice leaf disease diagnostic technique. It begins with the creation of a new training data, then progresses through the creation of an emergent Conn structure, deep extract for training the model, and lastly, detection of rice leaf illnesses. The whole thing.[Mead, 2007]

- The procedure is separated into steps, and the data is limited.

\section{LITERATURE REVIEW}

In this review presents a high-level overview of the main image-based machine learning techniques utilized in rice disease detection. A recent examination carefully assessed and analyzed various initiatives in the same direction. A recent research has performed a complete analysis and evaluation of cotton leaf diseases in a comparable arena.

\section{P. Regather (2011)}

In this paper research on the relevance of data mining approaches in agriculture has been given. Decision techniques such as C4.5, SVM deep learning and $\mathrm{ABC}$ algorithm have been shown in the field of agriculture. For the first time in agricultural history, data mining is being used to anticipate agricultural crop/animal/soil management via the use of data. In this research,data mining may be used to agriculture and other related fields.

\section{Jitesh P. Shah (2016)}

In This paper Images of disordered rice crops may be used to diagnose rice plant ailments using image processing and machine learning techniques is used. There is a lot of information in this article on various methods of diagnosing plant diseases, but there is also a straightforward explanation of the basic concepts behind picture processing and analysis. Based on the essential criteria, I perform a comprehensive assessment of 19 articles, which include research on rice plant diseases and other plants and fruits. These criteria include the amount of the dataset, the number of categories (diseases), pre-processing, segmentation methodologies, classifier types, classifier performance, and etc. Rice grain detection of disease might benefit from our survey and study. Studies on how to detect disease from plant photos is an exciting one for computer and agricultural researchers alike.

\section{Harshadkumar B (2017)}

In This paper demonstrates how to identify and classify rice infections using images of sick rice crops as a prototype technique is $\mathrm{i}$ am using. This prototype system was created after a thorough investigation of various image processing techniques. Rice is affected by diseases such as leaf blight, leaf spots, and leaf smut. Using a digital camera, I photograph diseased rice plants in a rice field. Four different approaches to reducing background noise and three different approaches to segmentation are tested. In order to ensure accurate feature extraction, I propose a centroid trying to feed K-means cluster method for disease component separation from a leaf photo. The author improves the K-means clustering results by removing the green pixel from the sick section. Color, form, and texture are the three types from which a variety of different features can be extracted. SVM is used for multiclass classification (SVM) and achieves 93.33 percent accuracy on the training dataset and 73.33 percent accuracy on the test data. Another method I employ is cross-validation, which has an accuracy rate of 83.80 percent and 88.57 percent, respectively. Machine learning principles from the computing industry could be used to detect plant diseases in images. This is an intriguing new area of agricultural research.

\section{Atal Patel, PhD (2018)}

In this paper reviews as far as understanding goes, the mythology utilized to diagnose illnesses, divide affected areas, and classify diseases. Are view of several feature extraction, segmentation, and classification methods, as well as their advantages and disadvantages, is also provided. Every country's economic success depends on the quality of its agricultural produce. Thus, it is possible to reduce crop loss and production losses by detecting the dangerous zones of plants. For disease classification prior to this new method, it needed a lot of time and effort and on-going farm surveillance. Technology improvements and a focus on this issue have made it possible to come up with an ideal answer in recent years. Machine learning, image analysis, and classification technologies have all been used it to identify and diagnose illnesses on agricultural produce. This research examines a wide range of methods for determining whether a food product is infected. Reviews Mythology is employed for disease diagnosis, division of the affected area, and disease classification. Features extraction methods, segmentation techniques and classifiers are also discussed in this article. Every country's economic success depends on the quality of its agricultural produce. The ability to identify areas of a plant that are potentially damaging might thus be considered a preventative measure against crop losses and productivity declines. Time and effort were necessary to use the former standard method for illness detection and classification. Technology developments and a focus on this issue have 
made it possible to come up with the best answer. Machine learning, image analysis, or classification technologies are used to identify and diagnose illnesses on agricultural goods. This research examines a wide range of methods for determining whether a food product is infected.

\section{Ronnel R. Atole (2018)}

This studygives how a machine learning technique is used to categorize rice plants depending on the health of its leaves. A3 classifier for normal, ill, and caterpillar plants was created using a learning method derived from such a Fully-connected convolutional model. The network achieved an effectiveness of $91.23 \%$ using learning algorithm with a modest sample length of 30 (30) or a learning cycle rate of 0.0001 . There were 600 images of rice plants used in the training, each representing a different class. Researchers used rice fields near here to gather our data, which was then checked by local farmers.

\section{A A JE Veggy Priyangka (2018)}

In this research as a result of the results, the accuracy rate was 95.24 percent. An agricultural-based population is common in Indonesia. An abundance of land and a tropical climate aid Indonesia's agricultural business. Indonesia's agriculture industry includes the production of rice. The amount of rice produced in Indonesia has decreased each year. Because of this, rice production factors are critical. The decline in Indonesian rice production is partly due to crop diseases. Rice plant infections are easier to spot because to technological improvements. Machine learning is one of the methods utilized to identify many types of rice ailments. Rice plant diseases were classified using a Fully Convolutional method. Machine learning technique known as Dnn $(\mathrm{CNN})$ is used to identify things. The VGG19 design is a good fit for this method since it offers features that improve results. There are 105 shots in the picture used for training and testing that are divided into training phase pictures. Data augmentation and period modifications may be used to test parameters.

\section{SantiKumariBehera (2018)}

This paper presents orange fruit deformation detection and classification using a machine vision-based system the seriousness of the problem is indicated by the symptoms, and Author recommend the best treatment. Additionally, it is critical to precisely identify the problem before it has a substantial impact and to give appropriate treatment. To minimize environmental harm and economic suffering, pesticides must be used in a precise amount based on the severity of the sickness. Author used a classifier SVM with Kmeans clustering for sickness classification with 90percentage accuracy and fuzzy logic to assess the level of disease activity.

\section{Md. JahidHasan (2019)}

In this paper, $\mathrm{An} \mathrm{AI}$ model termed deeper $\mathrm{CNN}$ was utilized in combination with SVM. The transfer learning method was used to strengthen the proposed model. This proposed model is then retrained using 1080 images from our database of nine unique rice diseases. Classifier is built utilizing characteristics extracted from the Deeper CNN model. As much as $97.5 \%$ of the time, the proposed method properly identified and classified nine unique types of rice disorders. Rice is one of the world's most often eaten foods. Farmers, on the other hand, are still unable to grow rice in the quantity planned due to a range of rice ailments. As a consequence, the quality, quantity, and production of rice are all adversely impacted. "A dearth of knowledge and a paucity of physicians with the necessary training make it difficult for farmers to diagnose illnesses. Detecting and categorizing rice infections manually takes a lengthy time as well. As a consequence, the need for an automated and exact identification has increased. Artificial Consciousness or high-quality computer vision algorithms have been used to develop an innovative and lucrative method for identifying rice diseases.

\section{Sherly Puspha Annabel (2019)}

This paper provides an introduction to several types of plant ailments as well as different machine learning methods for identifying diseases in different plant leaves. Lents are considered important because they provide people with energy. Plant diseases can damage the leaf at any stage between sowing and harvesting, resulting in significant crop losses and market value. According to my calculations, As a result, diagnosing leaf diseases in the agricultural sector is critical. However, a large number of workers, a longer processing time, and a thorough understanding of plant diseases are all required. As a result, machine learning is frequently used to diagnose plant diseases because it analyses data from multiple perspectives and assigns it to one of a predetermined set of categories.

\section{Benjamin Doh (2019)}

According as per I suggest paper suggests a better and more recent method for diagnosing fruit infections by using physical properties. As possible solutions, I suggested the KMeans clustering algorithm, the ANN, and the SVM. KMeans is a method for segmenting images. It assigns photographs to disease categories based on phenotypic features such as texture, color, whole architecture on the fruit, or physical make-up. When it comes to improving classification or detection accuracy, using ANN (Artificial Neural Network) over other algorithms has several advantages. In comparison to other image categorization techniques, they require very little pre-processing. Perhaps the network looked into hand-engineered filters from the past. It is a significant advantage to be able to create features without relying on prior knowledge or human effort. To improve classification accuracy, a SVM can be used in conjunction with an ANN. A new method for identifying and classifying fruit infections has been proposed. Fruit infections may have a negative impact on agricultural output and profitability all over the world. Fruits are becoming increasingly important in a healthy diet, according to a growing body of research. If you're trying to eat a healthy diet, fruits should be your starting point.

\section{T. Daniya (2019)}

In This paper Using different ML and image processing techniques, I concentrate mostly on disease diagnosis in rice plants based on images of ill plants. ML and image processing concepts are also discussed in the identification and classification of plant diseases. PNN, Algorithms, k-Nearest Neighbor Classifier, and Support Vector Machines are some of the classification methods used in agricultural research (SVM). Choosing a classification method is critical since diverse input data yields various quality results. For example, biological science and agriculture both employ plant leaf disease categories. This study covers all aspects of rice plant sickness, including the amount of the dataset, preprocessing, segmentation, and classification methods. In order to 
minimize a decrease in agricultural output quantity and production, disease identification in plants is essential. Agricultural challenges may be alleviated using various machine learning and image processing techniques are used.

\section{Vimal Shrivastava (2019)}

The most common methods used in traditional approaches are laboratory testing and eye examination. Because of a lack of understanding, an error may be made during a visual examination, and the testing technique is time-consuming but may not provide results on time. As a result of these factors, a new plant disease detection and classification method based on visual machine learning has been developed. The disease of the rice crop (Oryza Sativa) is the focus of this research. Images of leaves and stems showing signs of illness were captured in a rice field. My collection of 619 damaged rice grain images included Rice Blast (RB), Bacterial Leaf (BLB), Sheat Blight (SB), and Healthy Leaves (HL) (HL). As a feature representation, I used CNN architecture $(\mathrm{CNN})$, and as a classifier, I used a Vector Support Machine (SVM) (SVM). My research yielded positive results. This technique of early diagnosis of rice diseases could be used in conjunction with each other as a preventative measure and an alarm system. It could also be used to detect rice leaf disease in an agricultural setting. Early disease detection is one of the most important aspects of crop security.

\section{Inlay Antara (2020)}

The paper describes order to identify plant disease, proposed a prediction method that incorporates machine learning techniques. Processing a leaf's raw image and extracting properties such as its shape and color is the first step in the process. A variety of machine learning algorithms are used to categories the leaf image. Results are compared with Random Forest, Sim, Neighbor, and Artificial Neural Network. The proposed random forest classifier performs well when compared to current classifiers. One of most essential pillars of the Indian market is agriculture. People who live in rural regions are heavily dependent on agriculture for their livelihood. One of the key causes of lower crop production is a disease that affects plant growth. The discovery of remedies that may decrease the spread of the disease on plants is helped by early identification of sickness. One of the best ways to identify plant illnesses is to check the leaves. Computer vision and machine learning are evolving technologies that allow computers to recognize and analyses data in digital images.

\section{Striped Swain (2020)}

This study of Plant disease and it is a term used to describe the appearance of distinct patterns on plant leaves. It is thus possible to avert a decrease in productivity and agricultural production by recognizing ill plant zones. It is crucial for disease diagnoses and prevention to identify plant diseases caused by viruses, bacterium, fungi, and other microbes at an early stage. It takes a lot of time to do manual sickness detection. As a consequence, experts in the field are required to identify the problem. Conventional methods such as different classifiers, image processing and machine learning methods are utilized to detect and diagnose illnesses on agricultural yields. An overview of current methods for detecting disease in agricultural goods is presented here. Other classification methods and their analyses are also included in this review of leaf disease classification and diagnosis. Segmentation, feature extraction, and several classification techniques are also covered. The agricultural production of India is widely recognized among farmers. All nations depend on agricultural products for their economic well-being. The quality and quantity of agricultural products are reduced by plant diseases.

\section{Joaquim Filipe (2020)}

In this paper, using an in-depth investigation of the history of the QTP idea in IR, Author developed an original framework for assessing its applicability. It is that the AQtpUIR system, which stands for Adapted QTP orientated user retrieval of Information, would improve the relevancy of the information that is returned to the user. In this scenario, the relevance estimate is a weighted blend of DT and QT statistics. According to this understanding of QTP's purpose, it is a tool for encouraging documents to be used in a way that encourages both discovery and balance. In this design research, author emphasis the appropriateness of QTP measures for reconciling several sought-after trade-offs, such as relevancy, novelty, \& outcome variation (Coverage and Topicality). The information system relies heavily on file statistics, such as the frequency distribution (tf), the inverse documents (idf), and so on, to demonstrate relevance. For assessing relevance in information retrieval, Present Sample Distance (QTP) inside the matched texts is underutilized.

\section{N Gobalakrishnan (2020)}

In This Paper shows these ideas of a comprehensive investigation of several image-based plant diseases. Infecting any part of the plant, these diseases may be devastating. As a consequence of these ailments, which diminish agricultural productivity and increase economic expenses, I've suffered significant cumulative losses. It is imperative that the agricultural industry be capable of withstanding and growing inside the face of these obstacles. Monitoring a plant's health or diagnosing sickness at the proper time may help achieve this goal. Although technology has spread its wings to every facet of our everyday lives, it has yet to have an influence on agriculture. The agriculture industry is thriving despite the use of outdated technology. Crop disease misdiagnosis may have a substantial impact on productivity, time, cost, or product quality. To guarantee successful cultivation, it is necessary to keep an eye on the plant's progress throughout its life cycle. Researchers have been employing image processing techniques to monitor and detect plant diseases at various stages as a technical improvement. Many $\mathrm{ml}$ algorithms are being developed and deployed to correctly diagnose and treat a wide range of ailments on crops throughout their life cycles. Farming strategies that can predict the diseases that will affect plants throughout their lifetimes might be very beneficial.

\section{Dengshan Li (2020)}

To Research I was faced with the challenge of learning incorporates feedback design with a unique backbone in order to finally create a genuine crop disease and pest video detection approach for recognizing plant illnesses in films. To detect using the still-image detector, I first converted the film to a still frame and then synthesized the images into video. In the detection of still images, I used the faster-RCNN architecture. Image-training methods can be used to detect grainy films. To demonstrate the quality of information retrieval during the trials, additional clip evaluation criteria based on the classifier were proposed. Our algorithm with the modified backbone outperformed VGG16, ResNet-50, ResNet-101, and Classifiers for the detection of untrained rice videos. The amount of grain produced has a direct impact on a 
country's ability to feed its people. Controlling agricultural pests in a timely manner should result in increased grain production.

\section{Dr. Sala Krishna Rao (2020)}

Rice illnesses classified and detected in a novel way in this paper. This included rice blasting, rice tungro, and a fictitious version of smut disease. Several elements of the image segment, such as its shape and color, were extracted using an algorithm. After extracting all attributes, SVM (Support vector Machine) or k-Nearest Neighbor $(\mathrm{kNN})$ were used to identify illnesses $(\mathrm{k}-\mathrm{NN})$. Furthermore, the authors of this paper propose a cloud-based cooperative network that provides plant disease diagnosis to growers. Customers can upload photographs of various areas of the leaf to a smartphone app to diagnose real-time plant problems. The Indian economy is heavily reliant on agriculture, which generates a substantial amount of revenue. Early detection of leaf diseases is critical for maximizing agricultural yield and profit. Most illnesses are identified by agricultural experts based on outward signs. Farmers have fewer professionals available to them than those in the service industry.

\section{Punitha Kartikeyan (2021)}

In this paper, I will provide an overview of current methods for identifying and classifying plant diseases. Agriculture employs more than $70 \%$ of the Indian population. Plant diseases have a negative impact on agricultural output and quality. Bacteria, fungi, and viruses cause the majority of plant diseases. Manually identifying and diagnosing leaf diseases on large farms is time-consuming and costly. Plant health monitoring and disease detection are major challenges in sustainable farming. As a result, there is a growing need to use image processing methods to diagnose plant disease earlier and more accurately. The procedure also includes illness categorization and feature extraction. Plant health must be monitored on a regular basis in order to optimize production quality and yield. The computerized image analysis system, for example, is a powerful tool that can detect problems much earlier than the naked eye. It allows farmers to take the necessary precautions to protect their crops and ensure that their agricultural yield meets their expectations. Plant diseases have been classified using classifiers such as Svms, Artificial Neural Networks, U t Neighbors, and many others.

\section{CONCLUSION}

Only a few researches have been done in India on the automated classification of rice illnesses. This was the first time that have deep learning of convolutional models was examined. I categorise rice plant diseases based on their severity. In addition, a set of training examples was used to divide the whole dataset into separate ratios for the tests. $80 \%$ of Rice diseases can be categorised by my proposed model, because there are no standardized labelled rice disease images, comparisons of the recommended model literature and studies are not appropriate. To improve the performance of the proposed model, a large collection of ill rice photographs might be used.

\section{REFERENCES}

[1] Mead, G. C. (2007). Microbiological analysis of red meat, poultry and eggs. 348 .

[2] Yousef, A. E. (2010). Analytical food microbiology.
[3] Kornacki, J. L. (Jeffrey L. (2010). Principles of microbiological troubleshooting in the industrial food processing environment. 193.

[4] Revathi, P., Revathi, R., \& Hemalatha, M. (2011). Comparative Study of Knowledge in Crop Diseases Using Machine Learning Techniques. International Journal of Computer Science and Information Technologies (IJCSIT), 2(5), 2180-2182.

[5] Taormina, P. J. (2012). Microbiological research and development for the food industry. Microbiological Research and Development for the Food Industry, 1-327.

[6] Prajapati, B. S., Dabhi, V. K., \&Prajapati, H. B. (2016). A survey on detection and classification of cotton leaf diseases. International Conference on Electrical, Electronics, and Optimization Techniques, ICEEOT 2016,2499-2506.

[7] Prajapati, H. B., Shah, J. P., \&Dabhi, V. K. (2017). Detection and classification of rice plant diseases. Intelligent Decision Technologies, 11(3), 357-373.

[8] Ganatra, N., \& Patel, A. (2018). A Survey on Diseases Detection and Classification of Agriculture Products using Image Processing and Machine Learning. International Journal of Computer Applications, 180(13),7-12.

[9] Behera, S. K., Jena, L., Roth, A. K., \&Seth, P. K. (2018). Disease Classification and Grading of Orange Using Machine Learning and Fuzzy Logic. Proceedings of the 2018 IEEE International Conference on Communication and Signal Processing, ICCSP 2018, 678-682.

[10] Srinivas, B., \&Rao, G. S. (2019). To Secure Your Paper As Per UGC Guidelines We Are Providing An Electronic Bar Code. 09(June).

[11] Sherly Puspha Annabel, L., Annapoorani, T., \&Deepalakshmi, P. (2019). Machine learning for plant leaf disease detection and classification - A review. Proceedings of the 2019 IEEE International Conference on Communication and Signal Processing, ICCSP 2019, 538-542.

[12] Doh, B., Zhang, D., Shen, Y., Hussain, F., Doh, R. F., \&Ayepah, K. (2019). Automatic citrus fruit disease detection by phenotyping using machine learning. ICAC 2019 - 2019 25th IEEE International Conference on Automation and Computing, September, 1-5.

[13] Daniya, T., \&Vigneshwari, S. (2019). A review on machine learning techniques for rice plant disease detection in agricultural research. International Journal of Advanced Science and Technology, 28(13), 49-62.

[14] Swain, S., Nayak, S. K., \&Barik, S. S. (2020). A Review on Plant Leaf Diseases Detection and Classification Based on Machine Learning Models SasmitaKumariNayak Swati SucharitaBarik. MuktShabd Journal, 9(6), 5195-5205.

[15] Gobalakrishnan, N., Pradeep, K., Raman, C. J., Ali, L. J., \&Gopinath, M. P. (2020). A Systematic Review on Image Processing and Machine Learning Techniques for Detecting Plant Diseases. Proceedings of the 2020 IEEE International Conference on Communication and Signal Processing, ICCSP 2020, 465-468. 
[16] Ganatra, N., \& Patel, A. (2020). A multiclass plant leaf disease detection using image processing and machine learning techniques. International Journal on Emerging Technologies, 11(2), 1082-1086.

[17] Li, D., Wang, R., Xie, C., Liu, L., Zhang, J., Li, R., Wang, F., Zhou, M., \& Liu, W. (2020). A recognition method for rice plant diseases and pests video detection based on deep convolutional neural network. Sensors(Switzerland),20(3).
[18] Kartikeyan, P., \& Shrivastava, G. (2021). Review on Emerging Trends in Detection of Plant Diseases using Image Processing with Machine Learning. International Journal of Computer Applications, 174(11),39-48.

[19] Shrivastava, V. K., \&Pradhan, M. K. (2021). Rice plant disease classification using color features: a machine learning paradigm. Journal of Plant Pathology, 103(1), $17-26$.

[20] WHO, F. A. O. (n.d.). Statistical Aspects of Microbiological Criteria Related to Foods. 